\title{
CLASSICAL AND QUANTUM PHYSICS IN SELECTED ECONOMIC MODELS
}

\author{
Ewa DRABIK \\ Faculty of Management \\ Warsaw University of Technology, Warsaw, Poland \\ e-mail: ewa.drabik@poczta.fm
}

\begin{abstract}
A growing number of economic phenomena are nowadays described with methods known in physics. The most frequently applied physical theories by economists are: (1) the universal gravitation law and (2) the first and second law of thermodynamics. Physical principles can also be applied to the theory of financial markets. Financial markets are composed of individual participants who may be seen to interact as particles in a physical system. This approach proposes a financial market model known as a minority game model in which securities and money are allocated on the basis of price fluctuations, and where selling is best option when the vast majority of investors tend to purchase goods or services, and vice versa. The players who end up being on the minority side win.

The above applications of physical methods in economics are deeply rooted in classical physics. However, this paper aims to introduce the basic concepts of quantum mechanics to the process of economic phenomena modelling. Quantum mechanics is a theory describing the behaviour of microscopic objects and is grounded on the principle of wave-particle duality. It is assumed that quantum-scale objects at the same time exhibit both wave-like and particle-like properties. The key role in quantum mechanics is played by: (1) the Schrödinger equation describing the probability amplitude for the particle to be found in a given position and at a given time, and as (2) the Heisenberg uncertainty principle stating that certain pairs of physical properties cannot be economic applications of the Schrödinger equation as well as the Heisenberg uncertainty principle. We also try to describe the English auction by means the quantum mechanics methods.
\end{abstract}

Key words: quantum mechanics, minority games, equilibrium, English auction.

\section{1}

\section{Introduction}

The application of the laws of physics in economic theory has become a prominent trend for several decades. Economists have most often referred to the law of gravitation, static physics and the first and second law of thermodynamics. Gravitation models were pioneered in the early 1900s by J. Q. Stewart who defined the concept of demographic force (as an equivalent of potential energy) and demographic potential based on the theory of the field of gravity. Gravitation models became popular in many areas of economic study in the early 1960 s, e.g. the description of interaction between economic centres or international trade exchange.

The rules of thermodynamics, which generally state that the increase of internal energy of a system is equal to the difference between the amount of energy delivered to the system and the amount of energy released by the system into the environment, have been applied in the theory of economic equilibrium which can be expressed in a number of ways. Equilibrium has been studied by classical economists such as A. Smith, A. Cournot, W. Pareto, and recently by J. Tinbergen,
P.A. Samuelson, J.R. Hicks, R. Selten, J.F. Nash, R. Lucas and others.

Static physics, which focuses on electromagnetic interactions, has been applied in the financial markets theory. A financial market is defined here as a system made up of a number of individual participants who interact with one another in an electromagnetic-like manner. This approach to financial markets developed a minority game market model, where assets (stocks and money) are allocated solely based on price fluctuations: if the majority of investors are willing to buy it is more profitable to sell and vice versa. Minority players are winners.

So far, we have looked at classical physical concepts applied in economic theory. This paper also attempts to demonstrate the benefits of applying quantum mechanics in economic modelling. Developed around 1926, quantum mechanics is a theory describing behaviours of micro-objects in the framework of its principal concept of wave-particle duality. Micro-objects are believed to be simultaneously characterised by a wave function and properties of elementary particles. 
The key formula of quantum mechanics is one developed by E. Schrödinger, which determines the probability of a micro-object's being in a specific state. The other cornerstone of the theory is the Heisenberg uncertainty principle which states that no two different physical quantities of a studied object can be determined with any accuracy at the same time. This paper attempts to identify possible economic applications of both the Schrödinger equation and the Heisenberg principle. Special attention is given to a quantum model of English auction.

The structure of this paper is as follows: Part Two reviews selected economic concepts where modelling is based on laws of classical physics such as gravity, the first and second law of thermodynamics and static physics; Part Three provides a brief description of quantum mechanics, including the presentation of the Schrödinger equation and the Heisenberg principle, and suggests possible applications in economics; Part Four presents an attempted description of the English auction using the laws of quantum mechanics.

\section{Review of economic applications of the laws of physics}

\subsection{Gravitation models}

Economic models that use the basic laws of physics have been known since the 1960s. The most frequently applied are the law of gravity and the first and second law of thermodynamics.

In physics, gravity is the property of matter which consists in a mutual attraction between material bodies. The theory of gravity, also known as the Newtonian mechanics, is believed to have been first proposed by I. Newton (1687). In the Newtonian (classical) mechanics, the mass of a material point is defined as a positive scalar value which is the measure of the point's inertia. It is agreed that the mass of an object is independent of its movement status (it is constant) and is not affected by any processes within it.

This is known as the law of conservation of mass. Force is defined as vector value that illustrates the measure of mechanical impact of other objects on the body at hand. This is the way object may but do not have mutually exchange energy. Energy is a physical scalar value which is the general measure of the various forms of movements of matter that are contemplated by physics. Classical mechanics observes the principle of relativity, which says that the principles of mechanics shall be identical for all inertia reference systems, i.e. all mechanical processes that occur in the same conditions shall progress identically.

The law of universal gravity is one of the most widely known laws of classical physics. It states that every two material points shall mutually attract with force which is directly proportional to the product of the masses of both points and reversely proportional to the square of the distance between them, i.e.:

$$
\mathrm{F}=\mathrm{G} \frac{\mathrm{m}_{1} \mathrm{~m}_{2}}{\mathrm{r}^{2}}
$$

where:

$\mathrm{m}_{1}, \mathrm{~m}_{2}$ - are the masses of material points,

$r$ - is the distance between such points,

$\mathrm{G}$ - is the proportionality constant,

$\mathrm{F}$ - is the force of gravity.

The gravitational force is not a quantity that characterises the gravitational field. The gravitational field is quantified by the gravitational field strength expressed as follows:

$$
\gamma=\frac{\mathrm{M}}{\mathrm{r}^{2}}
$$

where $M$ is the mass of the source.

Economic theory also applies the concept of potential energy, as described by the following formula:

$$
E_{p}=-G \frac{M m}{r}
$$

Potential energy reflects the work done when a body is moved from one level to another. The gravitational field is characterised by a potential expressed as follows:

$$
V=-G \frac{M}{r}=\frac{E_{p}}{m}
$$

Potential is a physical quantity that specifies energy properties of the gravitational field at one point.

The first attempts at applying gravitational models in spatial economic research were made in early $20^{\text {th }}$ century. However, the pioneering work in this field came from demographer J.Q. Stewart, who defined the demographic force concept as a counterpart to potential energy or gravitational field potential (1947). Proper gravitational models used in economic theory date back to the 1960 s. 
Material points were replaced by 'centres' such as cities, shopping centres and the term 'gravitation' was replaced by its economic equivalent: interaction. One of the initial models was expressed as:

$$
I_{i j}=G \frac{P_{i} P_{j}}{d_{i j}{ }^{c}}
$$

where:

$I_{i j}$ - means interaction (impact) between area $\mathrm{i}$ and $\mathrm{j}$,

$\mathrm{P}_{\mathrm{i}}, \mathrm{P}_{\mathrm{j}}$ - are masses of two areas which can be measured by the size of their populations,

$d_{i j}$ - is the distance between area $i$ and $j$,

$\mathrm{G}$ - is the equivalent of gravitation force which is referred to as calibration constant in economics,

$\mathrm{c}-$ is the exponent of the power of the distance.

Units (masses) can be countries, economic regions, administrative provinces, cities or towns. Depending on the problem at hand, mass is measured in the number of households, number of consumption sites, number of warehouses and outlets, surface of retail or storage space, number of hospital beds, newspaper and magazine circulation, population etc. Distance is measured in kilometres, time of travel or the quotient of the product of price and time of travel. The calibration constant is model-specific and it is assessed so as to fit the model to the data.

There are a number of areas where gravitation models can be applied. For example, market researcher W. Reilly examined ways of assessing the sales proportion $\mathrm{s}_{\mathrm{i}}$ of the same goods offered by two mutually competing cities $M_{1}$ and $M_{2}$ to and smaller town $m_{0}$ located between them. He quantified the impact of city $\mathrm{M}_{\mathrm{i}}$ ( $i=1,2$ ) with mass $P_{i}$ (mass measured in population size) with distant by $\mathrm{d}_{\mathrm{i}}$ on goods available to town $\mathrm{m}_{0}$. The relationship $\mathrm{w}_{\mathrm{i}}$ is illustrated by the formula:

$$
\mathrm{w}_{\mathrm{i}}=\frac{\mathrm{P}_{\mathrm{i}}}{\mathrm{d}_{\mathrm{i}}^{2}}
$$

Impact $\mathrm{w}_{\mathrm{i}}$ refers to the concept of gravitational field. The sales ratio for two comparable cities is expressed as follows:

$$
\frac{\mathrm{s}_{1}}{\mathrm{~s}_{2}}=\frac{\mathrm{w}_{1}}{\mathrm{w}_{2}}
$$

which means that the value of sales in town $\mathrm{m}_{0}$ realised by the competing cities is proportional to their impacts.
By substituting values w1 and w2 (formula (6)) you get the following expression:

$$
\frac{\mathrm{s}_{1}}{\mathrm{~s}_{2}}=\frac{\mathrm{P}_{1}}{\mathrm{~d}_{1}^{2}}: \frac{\mathrm{P}_{2}}{\mathrm{~d}_{2}^{2}}=\frac{\mathrm{P}_{1}}{\mathrm{P}_{2}}\left(\frac{\mathrm{d}_{2}}{\mathrm{~d}_{1}}\right)^{2}
$$

Subsequently, the right-hand side of formula (8) is compared to 1 and then you quantify the reach of the competing market in the following way:

$$
\frac{\mathrm{s}_{1}}{\mathrm{~s}_{2}}=\frac{\mathrm{P}_{1}}{\mathrm{P}_{2}}\left(\frac{\mathrm{d}_{2}}{\mathrm{~d}_{1}}\right)^{2}=1, \frac{\mathrm{d}_{2}}{\mathrm{~d}_{1}}=\sqrt{\frac{\mathrm{P}_{2}}{\mathrm{P}_{1}}}
$$

If you accept that $d_{12}=d_{1}+d_{2}$ and you substitute the relevant values into formula (9) you get the 'optimum' distances of both cities from town $\mathrm{m}_{0}$ :

$$
d_{1}=\frac{d_{12}}{1+\sqrt{\frac{P_{2}}{P_{1}}}}, \quad d_{2}=\frac{d_{12}}{1+\sqrt{\frac{P_{1}}{P_{2}}}}
$$

'Point' $d_{12}$ is the point of separation between the two trading centres.

Here is another interesting model that determines trade exchange mechanisms between two countries. P. Krugman [5] uses the following equation to quantify trade between two countries:

$$
T_{i j}=\frac{A Y_{i} Y_{j}}{D_{i j}{ }^{c}}
$$

where:

A - is a constant,

$T_{i j}$ - means the value of trade between country $i$ and country $\mathrm{j}$,

$D_{i j}$ - is the distance between country $i$ and $j$,

$Y_{j}$ - is the GDP of country $j$,

c - is exponent of the power of distance.

Formula (11) entails that the value of trade is directly proportional to the product of GDP of the countries at hand and it decreases as the distance increases. The gravitational model demonstrates that trade between two countries is dependent on the size of their economies and is negatively affected by the distance between them. Other gravitational models describing spatial economic interactions were proposed by W. Reilly, M. Cadwallader, R. Bachi, T. Stanley, M. Sewall, P.D. Convers, D.L. Huff (Balicki [1]). 


\section{2 The law of conservation of energy and the theory of economic equilibrium}

Another example of fundamental laws of classical physics that have found applications in economic theory and social science is that of the general law of coservation of energy and the first law of thermodynamics. The law of conservation of energy says that the energy of a body or a system of bodies cannot come from nothing or disappear without a trace. One type of energy may merely convert into another and the total energy in an isolated system remains constant: $\mathrm{E}_{\mathrm{c}}=$ const.

The first law of thermodynamics, independently formulated by J.R. Mayer in 1842 and H.L.F. Helmotz in 1847 , is the law of conservation of energy proper in thermodynamics and it says that the internal energy of a system is solely the function of its state, i.e. is solely dependent on parameters that characterise the state of the system's equilibrium. It does not depend on the processes that have caused the system to be in that state. The increase of the system's internal energy is equal to the difference between the energy supplied to the system and the energy released by the system into the environment.

These laws have for many years been nearly intuitively applied in economics, mainly for describing economic equilibrium. The concept of economic equilibrium is ambiguous. Most commonly it is used to describe the balance between supply and demand for all goods (general equilibrium) or specific goods (partial equilibrium). It is widely accepted that economic systems are subject to numerous forces (often disturbing) but their overarching property is that they always tend toward equilibrium which is not necessarily the same as in the past.

The history of the concept of economic equilibrium goes back to the $17^{\text {th }}$ century. Adam Smith (1723 1790) in his groundbreaking work entitled "An Inquiry into the Nature and Causes of the Wealth of Nations" stated that an 'invisible hand of the market' (force) causes interactions between participants of an unbridled economy, or free market, to be harmonised, which then leads toward a market equilibrium. Thus, he introduced the concept of natural equilibrium. Around the same time, F. Qusnay (1694 - 1774) presented the Economic Table outlining the flows of value between the branches of economy, or an economic system which is subjected to certain economic factor but is in equilibrium. A. Cournot (1801 - 1877) pioneered the use of functional relationships in research of economic developments, which helped identify the differences between partial and general equilibrium. L. Walras (1834 - 1910), the creator of the price theory, applied the general equilibrium system to state that there is a close interdependency between the capital market, prices and the markets for products and services and all of the above affect each other. Any growth noted on any of the markets is reflected in the decreased potential of another, just like in a thermodynamic system. W. Pareto (1848 - 1923) claimed the existence of a lasting equilibrium to which economic systems return, favourable circumstances permitting, which offers a clear analogy to the law of conservation of energy.

Equilibrium and the 'forces' affecting economic systems can be expressed in a number of ways in economic theory. Originally, it was believed that an economy in equilibrium could be described with linear equations and inequalities. Underlying to this view was an assumption that equilibrium is preconditioned by a steadily growing economy. In such case, finding point (vector) of equilibrium $\mathrm{X}_{0}$ would require solving system of equations $\mathrm{AX}=\mathrm{B}$ and inequalities $\mathrm{X} \geq 0$ with a specified function of objective $f$ (criterion). The value of $\mathrm{X}_{0}$ is selected so that the objective function reaches the least value of all the solutions representing acceptable decisions. Such a minimum is naturally associated with equilibrium as does the minimum position of the pendulum in physics.

Over time, it was observed that the economic reality, as indeed physical reality, is fairly 'non-linear'. Nonlinear systems are more difficult to analyse than linear ones. The economic reality can be described using a system of non-linear differential equations and each object can have several positions (points) of equilibrium. Some of such points will be points of stable equilibrium and some will be short run, which means that there may be a position of equilibrium both with a minimum potential and kinetic energy.

The stability of the point of equilibrium further depends on the forces (distortions) operating against a specific economic object trying to throw it out of equilibrium. They may also change the position of the equilibrium point. Generally, the search for equilibrium in nonlinear dynamic systems requires that the evolving trajectory is defined. In specific cases, it may act chaotically. If this happens equilibrium can only be found 
by identifying the attractor and the attractor may turn out to be strange (Schuster [8]).

\subsection{Financial market modelling using minority games}

The last decade saw the rise in popularity of a financial market model called the minority game, which operates in a similar fashion to a macroscopic system in static physics. Market participants whose numbers are high (they are often referred to as a 'continuum') buy and sell securities. They rely on the analysis of the stock price fluctuation and other information they receive. If the majority of market participants intend to buy a stock it makes sense to sell, and vice versa. Minority group participants usually win. All market participants make decisions based on their experience and certain information patterns. There are three types of participants in a minority game depending on their response to available information: players who create information noise and make random decisions, producers who behave in a deterministic way and speculators who seek profit using all methods available. The activities of all three groups cumulate and cause qualitative change

of the entire system, as is the case in typical macroscopic physical systems where electromagnetic impacts are observed in the macro scale. Here, it is unnecessary to understand the impacts between elementary particles that make up the system because they are too small. From the point of view of a market participant it is essential to identify such parameters of the system which characterise the system's status in a macro scale. Because there are large numbers of participants, the laws of large numbers are often used (Challet et al. [2], Mosetti et al. [7]).

Minority games may be described as follows:

1) The financial market is assumed to be a non-zero sum game with $\mathrm{N}$ players $(\mathrm{N} \rightarrow \infty)$.

2) At any stage of the game $(t=1,2, \ldots)$, each player may but does not have to make one of two decisions: 'buy' or 'sell', which can be expressed as $a_{i}(t)=+1$ or $a_{i}(t)=-1$.

3) The payout (profit) of the i-th player is described by the following formula:

$$
g_{i}(t)=-a_{i}(t) A(t) \text {, where } A(t)=\sum_{j=1}^{N} a_{j}(t)
$$

$A(t)$ denotes the difference between the number of players who decided to sell and the number of players who buy a stock at time t. If $A(t)>0$ the players who play $a_{i}(t)=-1$ will win and those who chose $a_{i}(t)=+1$ will lose.

4) Strategy $s_{i}(t)$ of player $i$ at time $t(i=1, \ldots N ; t=1$, $2, \ldots)$ is assessed based on information $\{1, \ldots \mu, \ldots$ P \} obtained by players as the game proceeds. It is defined as follows: $\mathrm{s}_{\mathrm{i}}(\mathrm{t}):\{1, \ldots \mu, \ldots \mathrm{P}\} \rightarrow\{-1 ;+1\}$. In this case, information includes both the game history, or a string of minority and majority players' decisions in the past and certain information received from outside.

5) While considering both the received information and the 'adaptability' of individual players the profit of the $i^{\text {th }}$ player can be expressed as follows:

$$
g_{i}(t)=-a_{i}(t)_{s_{i}(t), i}^{\mu(t)} A^{\mu(t)}(t)
$$

where

$$
A^{\mu(t)}(t)=\sum_{j=1}^{N} a_{s_{j}(t), j}^{\mu(t)}(t)
$$

where

$$
\mathrm{a}_{\mathrm{i}}(\mathrm{t}) \rightarrow \mathrm{a}_{\mathrm{i}}(\mathrm{t})_{\mathrm{s}_{\mathrm{i}}(\mathrm{t}), \mathrm{i}}^{\mu(\mathrm{t})} \text { and } \mathrm{A}(\mathrm{t}) \rightarrow \mathrm{A}^{\mu(\mathrm{t})}(\mathrm{t})
$$

6) The goal of each market participant is profit maximisation $\mathrm{g}_{\mathrm{i}}(\mathrm{t})(\mathrm{i}=1, \ldots \mathrm{N})$ at any stage of game $\mathrm{t}$.

The problem can solved in a number of ways (Challet et al. [2], Mosetti et al. [7]). Financial market researchers who concentrated on minority game modelling have also focused on examining function $\mathrm{A}(\mathrm{t})$. Variance $\sigma^{2}(t)=\langle A(t)\rangle$ of quantity $A(t)$ measures the distribution of financial resources in the market. In addition, researchers study:

$$
\alpha=\frac{\mathrm{P}}{\mathrm{N}}
$$

When $\alpha>>1$ there is too much information circulating in the market and players act randomly. When $\alpha \rightarrow 1$ more players join the game and the information received is used more efficiently. When $\alpha$ reaches its minimum

$$
\left(\alpha=\frac{7}{20}\right)
$$

the behaviour of market participants can be compared to the description of critical phenomena in static physics. 
The probability of each player making a specific decision is determined as follows:

$$
\text { Prob }\left\{\mathrm{a}_{\mathrm{i}}(\mathrm{t})= \pm 1\right\}=\frac{\mathrm{e}^{ \pm \mathrm{g}_{\mathrm{i}}(\mathrm{t})}}{\mathrm{e}^{\mathrm{g}_{\mathrm{i}}(\mathrm{t})}+\mathrm{e}^{-\mathrm{g}_{\mathrm{i}}(\mathrm{t})}}
$$

Notably, minority games are based on a classic binary game called 'bar' (El'Farol bar problem) invented by W.B. Arthur where participants are obliged to make a decision each night whether or not to go to a bar.

The economic models presented thus far 'apply' the laws of classical physics. The following chapter presents the use of quantum mechanics in modelling economic phenomena.

\section{The application of quantum mechanics in economics}

\subsection{Basics of quantum mechanics}

Quantum mechanics is a theory describing the behaviour of elementary particles in atomic scale. The biggest accomplishment of quantum physics is the waveparticle duality. According to this theory, photons and electrons, light and matter are both waves and particles. An object such as an electron is a particle because of its spatial delineation and a wave because the way it moves in space. The following discussion will use the term micro-objects instead of particles.

Quantum mechanics says that nature can be described in approximated terms and each measurement is biased with a probability and accuracy. Uncertainty and inaccuracy are not caused by the inaccuracy of measuring equipment but are inherent to the phenomena themselves. It should be underlined, however, that quantum physics is characterised by an absolute determinism but exclusively in the space of states. Indeterminism starts when you move to the physical space and ask about the coordinates of a micro-object.

The major accomplishment of quantum mechanics is that it has rejected the classical division into the observed and the observer. In classical physics, the observer cannot influence the state of an object under observation or measurement. In quantum mechanics it is acceptable to assume that micro-objects may be located in several places at the same time by the time of observation. Also, they may be so strongly linked (quantum entanglement) that they may act as unity regardless of the distance between them. This property has its economic analogy. For example, strongly linked subsidiaries of a major corporation may be located in different parts of the world. Despite the distance between them they form one big 'organism'.

The fundamental concept of quantum physics is a space of states of physical systems that forms a vector Hilbert space $^{1}$. The state of a system is represented in quantum mechanics by unit vectors. That is known as the pure state. The scalar product is used for assessing the probability of measurement of any physical quantity.

Each physical quantity is additionally represented using a Hermitian operator ${ }^{2}$. The values of the operator are real numbers and this is why they are not interpreted as quantities to be obtained through measurement. After the measurement is taken, the system is in one of the Hermitian operator's own states which represents a given observable, as outlined by J. von Neuman. The case described by von Neuman involves a deterministic, i.e. predictable, evolution of the system. Physicist R.P. Feynman claimed that measurement has a purely random character. In this approach, it is possible to determine the probability of a certain value of the measured quantity. However, it is not possible to provide an accurate indication of the state of the system; you can only calculate mean values of 'specific observables' or determine the probability of such system moving to a certain state. Feynman stated also that the system with the highest probability moves along a classical trajectory, i.e. the one postulated by von Neuman.

According to the fundamental concept of quantum mechanics, i.e. the wave- particle duality, each microobject is both characterised by a wave function and by particle-specific parameters such as mass that can be located in a particular state. Beside location, microobjects are further characterised by quantum numbers that describe the micro-object's energy states. The main quantum number $\mathrm{n}=1,2,3, \ldots$ specifies stationary energy states static; orbital quantum number $1(1=0,1$, 2 ...) describes the micro-object's momentum; magnetic quantum number $\mathrm{m}(\mathrm{m}=0, \pm 1, \pm 2 \ldots)$ specifies the orientation of the micro-object's space; the spin quantum number describes the micro-object's intrinsic an-

\footnotetext{
${ }^{1}$ The Hilbert space is a unitary and complete space on which a scalar product has been identified. The completeness is a guarantee that each Cauchy string of elements is convergent to the elements of that space; furthermore, completeness means that while transitioning from one state to another it is impossible to go beyond the states that belong to the space at hand.

${ }^{2}$ Operator $\mathrm{T}$ is called Hermitian if for each $\mathrm{x}, \mathrm{y} \in \mathrm{X}$, where $X$ is a Hilbert space, the following is true: $(T x \mid y)=(x \mid T y)$; (|) is a symbol for a scalar product.
} 
gular momentum. The spin is constant for specific types of elementary particles. For example, particles with a spin of $1 / 2$ are called fermions (electron, proton, and neutron) as opposed to bozons whose spins are equal to 1 (photons) etc. It must be added that no atom can have two or more particles with an identical configuration of the four basic quantum numbers.

One critical concept in quantum mechanics is the Planck constant. In classical physics, the energy of any system evolves continuously. This means its values can be anywhere near each other. In contrast, quantum mechanics allows energy to have only specific discrete values that are equal to the total amount of elementary portions of energy quanta $\varepsilon_{v 0}, \varepsilon_{v}=\mathrm{k} \varepsilon_{v 0}, \mathrm{k}=0,1, \ldots$ (Feynman et al. [4]).

The quantum theory is a generalisation of classical physics. It is a broader and more comprehensive theory applied in both in the realm of atoms and planets. It suggests that objects with great masses and but short wavelengths given the distances at hand can be examined in terms of their evolution using Newtonian mechanics or classical thermodynamics. Similarly, economic theory can assume that the space of goods or any other space composed of economic objects is, like matter, made up of small indivisible portions. These portions do not form regular networks but move in all possible directions (it could barely be argued that there is continuous evolution of the market prices of goods or stocks). The smallest unit of price change is one grosz, cent etc., or 0,01 of a currency unit (CU). Equally, it may be one gram, piece etc. Economic systems, including the 'small' ones, are subject to market cycles with their own length and amplitude, which makes them a perfect analogy to waves.

\subsection{The Schrödinger equation and its applications in economics}

Another key concept in quantum mechanics is system evolution. Systems can evolve in two ways. First, deterministic evolution as described by a unitary operator: states of the system change in a predictable manner. Secondly, random evolution upon measurement: the only prediction that can be made is the probability of the system finding itself in a given state. The wave function plays a critical role in quantum mechanics. Let $\Psi(\mathrm{x}, \mathrm{y}, \mathrm{z}, \mathrm{t})$ be a wave function where $\mathrm{x}, \mathrm{y}, \mathrm{z}$ are location coordinates of an object in space $\mathfrak{R}^{3}$, and $\mathrm{t}$ is time. The square of function $\Psi$ specifies the probability of the micro-object located at point with coordinates $(\mathrm{x}, \mathrm{y}, \mathrm{z})$ at time t. Formally, $|\Psi|^{2}$ meets the following condition:

$$
\Psi^{*} \Psi=|\Psi|^{2}=\mathrm{p}
$$

where:

$\Psi^{*}$ - is a dual wave function of $\Psi$,

$\mathrm{p}$ - is probability.

Effectively, it can be demonstrated that the probability of the micro-object finding itself in an element with volume $\Delta \mathrm{V}=\Delta \mathrm{x} \Delta \mathrm{y} \Delta \mathrm{z}$ of space equals:

$$
\mathrm{P}=\mathrm{p} \cdot \Delta \mathrm{V}=\mathrm{p} \cdot \Delta \mathrm{x} \Delta \mathrm{y} \Delta \mathrm{z}
$$

By definition, the integral of the density of the probability throughout the volume of the space holding the object will equal 1:

$$
\int_{\mathfrak{R}^{3}}|\Psi|^{2} \mathrm{dV}=\iiint_{\mathfrak{R}^{3}}|\Psi|^{2} \mathrm{dxdydz}=1
$$

This, a wave function allows you to determine the probability of objects taking specific positions in a space. Consequently, it does not make sense to look for objects in locations where the square of the 'probability wave' amplitude modulus equals 0 . Microobjects will normally take fuzzy positions in a given portion of space and, since there are an infinite number of points in any small area, the probability assigned to each one of them cannot be a finite number (Feynman et al. [4]). In addition, the wave function provides a solution to a differential equation called the Schrödinger equation, expressed as follows:

$$
\mathrm{ih} \frac{\partial \Psi}{\partial \mathrm{t}}=-\frac{\hat{\mathrm{h}}}{2 \mathrm{~m}} \bar{\Delta} \Psi+\mathrm{U}(\mathrm{x}, \mathrm{y}, \mathrm{z}, \mathrm{t}) \Psi
$$

where

$\Delta$ - is the Laplace operator, i.e.

$$
\bar{\Delta} \Psi=\frac{\partial^{2} \Psi}{\partial \mathrm{x}^{2}}+\frac{\partial^{2} \Psi}{\partial \mathrm{y}^{2}}+\frac{\partial^{2} \Psi}{\partial \mathrm{z}^{2}}
$$

$\mathrm{m}$ - is the mass of the micro-object, $\mathrm{U}(\mathrm{x}, \mathrm{y}, \mathrm{z}, \mathrm{t})$ is the micro-object's potential energy dependent on its position,

$\hat{\mathrm{h}}=\frac{\mathrm{h}}{2 \pi}, \mathrm{h}$ is the Planck constant $\left(\mathrm{h}=6,626 \cdot 10^{-34} \mathrm{~J} \cdot \mathrm{s}\right)$, $\mathrm{i}=\sqrt{-1}$ is the imaginary unit.

Additionally, it is accepted that function $\Psi$ is finite, unique and continuous. 
In case function $U$ is not dependent on time $\left(\frac{\partial U}{\partial t}=0\right)$ the equation takes the following form:

$$
\bar{\Delta} \Psi+\frac{2 \mathrm{~m}}{\hat{\mathrm{h}}^{2}}(\mathrm{E}-\mathrm{U}) \Psi=0
$$

where

$\mathrm{E}$ - is the kinetic energy of the micro-object.

The equation is solved by finding wave function $\Psi$ and energy value E. There is no relationship to the distribution of potential energy that may be interpreted to affect the micro-object.

Economic systems are observed to be subject to market cycles. Hence, it is possible to ascribe wave properties to them. Further, they have their own mass (e.g. population of a country), as discussed in Section 2. The length of a wave related to market cycles is usually comparable to 'units' describing the mass of an economic system. Consequently, applying the rules of quantum mechanics in economics seems logical. It must be added that while he developed his equation for the micro-world, Schrödinger looked for inspiration in the macro-world, specifically on the propagation properties of light waves. Quantum effects become relevant where the object-related wavelength is comparable to distances, which is often the case in economic systems. Wave function can prove useful in forecasting as it allows to determine the probability of a certain object finding its way into a given area $(x+\Delta x, y+\Delta y$, $\mathrm{z}+\Delta \mathrm{z})$.

The Schrödinger equations could be applied for making projections of economic phenomena which evolve chaotically and do not follow a uniform curve, i.e. the system's evolution trajectory is an attractor, an often strange one (see e.g. Schuster [8]). Forecasting future values of such phenomena is fairly challenging. Only short-term forecasting is possible for strange attractors. Hence, instead of short-term projections it seems to make more sense to calculate the probability of a specific system findings itself in a given area $(\mathrm{x}+\Delta \mathrm{x}, \mathrm{y}+\Delta \mathrm{y}, \mathrm{z}+\Delta \mathrm{z})$, and this is feasible using the wave function. This method may be seen as an alternative to existing forecasting methods which are not always correct and may generate major inaccuracies.

The Schrödinger equation helps identify an 'area' in which the object is most likely to find itself in the future. An economic version of the equation is not easy but possible to construct. First, an equivalent to the Planck constant must b identified. If you examine a price or money related problem, the Planck constant may be 1 grosz, 1 cent etc. Depending on the problem at hand, it may be 1 gram, 1 piece etc. Secondly, if the trajectory of the economic system's evolution behaves chaotically the attractor related that trajectory should be spatially located and current coordinates should possibly be determined for the object's location. It does not make sense to search for future values of the phenomenon at hand outside the attractor area. Projections may use the 'closest neighbour'.

Presenting the $\Psi$ wave function may be the hardest part. In fact, methods known in the world of physics may prove helpful in building such functions for economic models (see Feynman et al. [4]), p. 288-290 and 299-301). After you have dealt with the challenges of identifying all the variables contained in equation (11) you may take to trying to solve it, i.e. find the location of an area $(\mathrm{x}+\Delta \mathrm{x}, \mathrm{y}+\Delta \mathrm{y}, \mathrm{z}+\Delta \mathrm{z})(\Delta \mathrm{x}, \Delta \mathrm{y}, \Delta \mathrm{z} \rightarrow$ $0)$, for which the square of the absolute value of function $\Psi$ is the largest. Such location will correspond to the best forecast of an economic phenomenon under study.

\subsection{Schrödinger's cat paradox}

The loss of the ability to forecast events while they transit from one state to another was described by Schrödinger in 1935. He presented a case of cat trapped in a box in which it may die as a result of electromagnetic discharge or it may survive. There is a fifty per cent chance of survival. Schrödinger claimed that according to quantum mechanics the 'state' of a cat in a box, before the box is opened, would be defined as both composed of a cat which is alive and dead, which can be expressed as:

$$
\left.\left.\left|\Psi_{\text {cat }}\right\rangle=\alpha \mid \text { alive }\right\rangle+\beta \mid \text { dead }\right\rangle
$$

where

$$
|\alpha|^{2}+|\beta|^{2}=1
$$

After the box is opened (i.e. after a measurement of the system is taken) an external observer would see either a living or a dead cat. This thought experiment may be taken to a higher level by placing an internal observer in a secure corner of the box. The observer would see the situation from within and track the cat's state immediately after the discharge that leads to the reduction of the state $\left|\Psi_{\text {cat }}\right\rangle$ to one of its constituents: $\mid$ alive $\rangle$ or $\mid$ 
dead $\rangle$, which will express itself as the following states, respectively: |happy $\rangle$ or $|\mathrm{sad}\rangle$. On the other hand, the external observer describes the system (containing the internal observer) using a linear superposition:

$$
\left.\left.\left.\left.\left|\Psi_{\text {cat }}=\alpha_{1}\right| \text { alive }\right\rangle \otimes \mid \text { happy }\right\rangle+\beta_{1} \mid \text { dead }\right\rangle \otimes \mid \text { sad }\right\rangle
$$

where $\left|\alpha_{1}\right|^{2}+\left|\beta_{1}\right|^{2}=1$

The external observer will lead towards the reduction of the superposition only after the box is opened. In essence, the experiment points to the fact that while the cat is still a superposition of a dead-alive cat for the external observer (prior to opening the box) the internal observer is already happy at the cat's survival or sad at the cat's death.

\subsection{The Heisenberg uncertainty principle and major formalisms of quantum mechanics}

Another important law of quantum mechanics is Heisenberg uncertainty principle which says that it is not possible to determine two different physical quantities of a studied object at the same time with any accuracy. It is applied both in classical as in quantum physics, often in connection with the accuracy of measurement. In physics, measurement is process of interaction between the instrument and the object during which certain information about the properties of the latter is obtained. Measurement often affects the process and this can be used for assessing a number of phenomena.

The Heisenberg principle says that the product of errors (in the sense of standard deviation) while measuring two physical quantities is not bigger than the Planck constant (or its multiple). The following inequalities describe the principle:

$$
\Delta x \Delta p_{x} \geq \hat{h} / 2, \Delta y \Delta p_{y} \geq \hat{h} / 2, \Delta z \Delta p_{z} \geq \hat{h} / 2
$$

where

$\Delta \mathrm{x}, \Delta \mathrm{y}, \Delta \mathrm{z}$ - are the ranges of the object's locations,

$\Delta \mathrm{p}_{\mathrm{x}}, \Delta \mathrm{p}_{\mathrm{y}}, \Delta \mathrm{p}_{\mathrm{z}}$ - are projections of momentum onto coordinate axes.

Symbols $\Delta \mathrm{x}, \Delta \mathrm{y}, \Delta \mathrm{z}, \Delta \mathrm{p}_{\mathrm{x}}, \Delta \mathrm{p}_{\mathrm{y}}, \Delta \mathrm{p}_{\mathrm{z}}$ mean root means squares of the deviation of coordinates and momentum projects from their mean values, i.e.:
$\Delta \mathrm{x}=\sqrt{\left\langle\Delta \mathrm{x}^{2}\right\rangle}=\sqrt{\left\langle\mathrm{x}^{2}\right\rangle-\langle\mathrm{x}\rangle^{2}}$, where $\langle\mathrm{x}\rangle$ is a physical symbol of a mean quantity.

It follows from Heisenberg's relation (22) that the product of the uncertainty of the measured momentum and the micro-object's location is never smaller than the Planck constant. The uncertainty principle may further be applied to energy and time. If a microobject's energy is $E$ then the accuracy of energy measurement $\Delta \mathrm{E}$ will depend on the time of measurement $\Delta \mathrm{T}$, as in the inequality:

$$
\Delta \mathrm{T} \Delta \mathrm{E} \geq \hat{\mathrm{h}} / 2
$$

Inequality (23) says that the product of the uncertainty of the time an object remains in a certain state and its energy are not bigger than Planck constant. The energy of a micro-object which remains in a stationary state is precisely specified and it equals $\Delta \mathrm{E}=0$.

Similar relations are found in classical physics in the description of a macro-scale wave movement. In general terms, the product of the uncertainty of a pair of canonically coupled quantities (e.g. $\left[p_{x}, x\right],[T, E]$ are canonically coupled) is not smaller than Planck constant.

The uncertainty relations with respect to location and momentum stipulate that no object can simultaneously have accurately specified coordinates and related momentum constituents. This property means that the concept of a micro-object's trajectory in phase space is losing in importance as it is not described by any timeparameterised and 'strictly specified' line but rather by a fuzzy area. Similarly, the trajectory of a large number of economic phenomena evolves chaotically.

\subsection{Major Theories of Quantum Mechanics and Differences Between Classical Physics and Quantum Mechanics}

To summarise, here are the main formulations of quantum mechanics:

1) A state function must be used for the description of any system.

2) A physical system is in a certain state with a certain probability.

3) For each dynamic quantity a form of description is used and it is called a linear operator.

4) State functions fulfil a state-specific Schrödinger equation. 
5) Every physical object is characterised using a wave function. One property of the wave function is that its root square is the probability of the object at hand having a specific spatial location.

6) A quantum object exhibits all the properties of a particle (mass) and a wave (frequency); it is represented by states which can be characterised using quantum numbers.

7) Quantities that characterise a quantum object, for example energy, do not have a specific value at a certain point in time but are characterised by a set of possible own values which can be calculated using Schrödinger equation.

8) The uncertainty principle is a consequence of the lack of commutation of canonically coupled operators such as location or momentum.

Note:

- a commutator is an operator which gives an indication to what extent matrix multiplication or operations of operators fail to be communitative, i.e. $[\mathrm{A} \mathrm{B}]=\mathrm{AB}-\mathrm{BA} \neq 0$,

- in quantum mechanics, operators that define physical quantities (observables) do not have to be commutative.

To sum up, differences between classical and quantum physics are significant. The classical theory says specifically where a given micro-object is located at a given point in time, or what its trajectory is. However, there is no experimental proof or disproof of such calculations. The quantum theory does not say anything about a specific location of a micro-object in space. Instead, it makes a projection of the probability of the micro-object's location at a point in time.

The superiority of quantum over classical physics is that its projections are confirmed by experimentation. Furthermore, classical physics says that 'all things physical' can be measured with any accuracy. In contrast, the Heisenberg uncertainty principle can be interpreted as a formulation of limits to accuracy. Classical physical methods cannot be helpful in recreating reality because reality is more than a set of defined elements.

\subsection{Suggested applications of certain quantum mechanics principles in social sciences}

The uncertainty principle is a universal law which can be applied in a number of disciplines. We believe it can also be applied in social sciences. The observation of economic processes appears to be an insufficient method of measuring and quantifying such phenomena, let alone making decisions under such uncertain conditions. Researchers use various statistical methods and mathematical models based on such formalisms as neuron networks, genetic algorithms or the theory of chaos. These methods return approximate results. For example, the interpretation of statistical data is affected by the method of data collection, data quantification or thought experiment planning, sampling, eliminating distortions or uncontrolled variables and many other factors. Similarly, neuron networks, though capable of learning and generalising knowledge, are nothing but approximations. In this case, approximations are even greater and contradicting signals may generate contracting decisions.

On the other hand, genetic algorithms emulate evolution. They test certain rules and select a set of such rules which optimise a given function. In order to make predictions based on genetic algorithms it is essential that a large body of input data should be collected, which is not always feasible and mistakes can be made at a very early stage of a research project.

The theory of chaos relies on the principles of the evolution of deterministic systems which may behave chaotically in certain situations. They are normally nonlinear systems and most of them cannot be precisely described, particularly if they relate to economic situations. While it is possible to apply Takens' delay embedding theorem the use of another approximative method makes the uncertainty of decisions or conclusions even greater.

To sum up, data collection, statistical and predictive methods are equivalents of measurement in physics. Overlapping approximative results generate a number of errors which cumulatively lead to a bias which is not smaller than a certain constant quantity that characterises the phenomenon at hand. This constant may be interpreted as an economic constant equivalent to Planck constant. Indeed, it may be Planck constant under certain circumstances (see Section 4).

There are more general considerations with regard to the application of quantum mechanics formalisms. According to Stapp H., the universe, and everything in it, may be presented as a universal wave function which evolves in time according to Schrödinger wave equation and determines the probabilities of events. 
In other words, every alternative option is represented by an evolving wave function ${ }^{3}$.

Schrödinger equation is believed by many physicists to be a nearly ideal representation of quantum physical phenomena and can prove a good predictive tool in social sciences. Notably, Schrödinger construed his equation using existing laws of the macro- rather than micro-world. He used the properties of heat waves and focused on issues linked to turbulence. The equation has been applied on numerous occasions, e.g. in atomic and hydrogen bomb programs.

The following section will seek analogies between the classical auction model and quantum mechanics laws.

\section{Analogies between the rules of English auction and the laws of quantum mechanics}

Auctioning is one of the oldest method of exchanging goods and currency. They are economic mechanisms designed to effectively allocate goods and money through bidding. Auction rules are normally predefined, which means they can be interpreted as the rules of the game, the game being the auction itself. Normally, the winner of an auction is the buyer, a player who offers the highest price for the auctioned goods. Auctions are mainly used for non-homogenous and unconventional goods, services and other benefits.

There are many types of auctions and they are usually classified by the form of delivery and method of transacting business. There are oral and written auctions. Oral auctions include English auctions, where participants present their price bids in an ascending order. The auction ends when the price is so high that no other bidder can 'outbid' and the bidder offering the highest price wins.

Another oral type if the Dutch auction where the price of a good is effectively reduced until a buyer is found. This type of goods and money allocation is used by second-hand shops and for selling perishable goods. Written auctions include first and second dealed bid auctions. The first dealed bid auction, also known as first-price sealed-bid auction (FPSB) is a process where bids are place in writing, e.g. in sealed envelopes.

\footnotetext{
3 This is quoted after A. Scott (1999), Schody do umystu (Stairway to the Mind), WNT Warsaw. This book is not listed in references because it has not been used in this publication. However, it is entirely dedicated to applications of quantum mechanics in biology.
}

The highest bid wins and the price of goods paid by the buyer is equal to the bidding price. The second dealed bid auction, also known as Vickrey auction ${ }^{4}$, is a process where the highest bid wins but the price paid by the buyer is the second highest.

There are other types of auctions and new types are being developed. The auction theory is one of the most vibrant areas of research combining economic and games theory formalisms. New mathematical auction models are based mainly on the games theory (see Drabik [3]). These models utilise the classical probability calculus. However, it often proves insufficient in addressing the nuances of auctioning. Therefore, attempts will be made to describe auctioning in the language of quantum mechanics. The main focus will be to present analogies between auction rules and quantum mechanics laws.

Without loss of generality, it can be assumed that buyers act rationally, which means they will stop short of buying goods above a predefined resignation price (a price of their own choice). Furthermore, it can be comfortably assumed that "purchase" and "sale" are equivalent concepts because selling goods also means buying money.

Let us consider an English auction with $\mathrm{N}$ buyers (players $1 \ldots \mathrm{N}$ ) and one seller (player 0). Let $|\Psi\rangle_{\mathrm{k}}^{5}$ be the vector of Hilbert space uniquely identifying the strategy or state of the $\mathrm{k}^{\text {th }}$ buyer. Function $\Psi(\mathrm{x})=$ $\langle\mathrm{x} \mid \Psi\rangle$ defines the amplitude of the probability of wave function $\Psi$. According to quantum theory, as briefly summarised in Section 3 above, it is accepted that the root square of probability amplitude modulus $|\langle\mathrm{x} \mid \Psi\rangle|^{2}$ is the density of the probability of the measure of quantity $\mathrm{x}$ of random variable $\mathrm{X}$ representing the state of the system.

\footnotetext{
4 William Vickey received a Nobel prize in economics in 1994, inter alia for his work in the area of auction theory.

${ }^{5}$ Strategies $|\Psi\rangle_{k}(k=1, \ldots N)$ are elements of Hilbert space $\mathrm{H}$. Formula $\langle\mid\rangle: \mathrm{H} \times \mathrm{H} \rightarrow \mathrm{C}$, known as a scalar product for any $\Psi$, $\Psi ', \Psi{ }^{\prime}, \in \mathrm{H}, \lambda \in \mathrm{C}$ has the following properties:

1) $\langle\Psi \mid \Psi\rangle \geq 0$,

2) $\langle\Psi \mid \Psi\rangle=0 \Rightarrow \Psi=0$,

3) $\left\langle\Psi \mid \Psi^{\prime}+\Psi^{\prime \prime}\right\rangle=\left\langle\Psi \mid \Psi^{\prime}\right\rangle+\left\langle\Psi \mid \Psi^{\prime},\right\rangle$,

4) $\langle\Psi \mid \lambda \Psi\rangle=\lambda\langle\Psi \mid \Psi\rangle, 5)\left\langle\Psi \mid \Psi^{\prime}\right\rangle=\left\langle\Psi^{\prime} \mid \Psi\right\rangle^{*}$, where the asterik denotes entaglement.
} 
Since Hilbert space is the major formalism of quantum theory the probability amplitude should be standardised to 1 according to the following formula (Piotrowski [8]):

$$
\langle\mathrm{q} \mid \Phi\rangle_{\mathrm{k}}=\frac{\langle\mathrm{q} \mid \Psi\rangle_{\mathrm{k}}}{\langle\Psi \mid \Psi\rangle_{\mathrm{k}}}(\mathrm{k}=1, \ldots \mathrm{N})
$$

where

$$
\langle\Psi \mid \Psi\rangle=\int_{-\infty}^{+\infty} \Psi(x) \overline{\Psi(x)} d x
$$

Expression (24) defines a standardised amplitude to the strategy that defines the commitment of the $\mathrm{k}^{\text {th }}$ buyer to purchase the goods at price q. Expression:

$$
\left\langle\mathrm{p}_{\text {in }} \mid \Phi\right\rangle_{0}=\frac{\left\langle\mathrm{p}_{\text {in }} \mid \Psi\right\rangle_{0}}{\langle\Psi \mid \Psi\rangle_{0}}
$$

is the seller's (player 0) commitment which is to specify reservation price $p_{i n}$, i.e. to specify a price under which the goods must not be sold. Reservation price $p_{\text {in }}$ is a measurement concept.

According to quantum mechanics formalisms, the density of the probability of a specific value of a random variable which characterises the $\mathrm{k}^{\text {th }}$ auction participant (player $\mathrm{k}$ ) is equal to the root square of the modulus of a strategy standardised to one:

$$
\mathrm{P}_{\mathrm{k}}=\left|\Phi_{\mathrm{k}}\right|^{2}=\frac{\left|\langle\mathrm{q} \mid \Psi\rangle_{\mathrm{k}}\right|^{2}}{\langle\Psi \mid \Psi\rangle_{\mathrm{k}}}
$$

The auction is won by the $\mathrm{k}^{\text {th }}$ buyer if his/her price is the highest which makes the probability of reaching state $\Psi_{\text {out }}$ the highest as well. Thus:

$$
\frac{\left|\left\langle\mathrm{q}_{1} \mid \Psi\right\rangle_{\text {out }}\right|^{2}}{\langle\Psi \mid \Psi\rangle_{\text {out }}} \leq \ldots \leq \frac{\left.\left\langle\mathrm{q}_{\mathrm{k}} \mid \Psi\right\rangle_{\text {out }}\right|^{2}}{\langle\Psi \mid \Psi\rangle_{\text {out }}}
$$

The equality sign means that strategies of auction participants may be entangled. In quantum theory, entanglement means a certain form of a correlated quantum state of two or more particles or other quantum systems. In other words, entangled quantum states provide an indication of an existing correlation between states despite the lack of proximity. The assumption of a single buying and selling price in auction theory is equivalent to the entanglement of quantum phenomena.
Bidding is process of dynamic transitions from one state to another or bidding higher and higher prices by buyers:

$$
\begin{aligned}
& \left\langle\Phi_{\text {in }} \mid \Phi_{\text {out }}\right\rangle=\sum_{\mathrm{i}}\left\langle\Phi_{\text {in }} \mid \mathrm{q}_{\mathrm{i}}\right\rangle\left\langle\mathrm{q}_{\mathrm{i}} \mid \Phi_{\text {out }}\right\rangle, \\
& \left(\mathrm{q}_{1} \leq \mathrm{q}_{2} \leq \ldots \leq \mathrm{q}_{\mathrm{k}}\right)
\end{aligned}
$$

where $q_{i}$ means price bid by the $i^{\text {th }}$ buyer. It can be assumed they correspond to base states $\mathrm{e}_{\mathrm{i}}, \mathrm{e}_{\mathrm{i}} \mathrm{e}_{\mathrm{j}}=\delta_{\mathrm{ij}}$ and $\langle\mathrm{i} \mathrm{j}\rangle=\delta_{\mathrm{ij}}$.

Note:

According to quantum mechanics, a transition from state $\mathfrak{\aleph}$ to state $\mathfrak{I}$ may be expressed as a sum after the system of base states of the product of amplitudes of transition from state $\aleph$ to the following base states up to state $\mathfrak{I}$ :

$$
\langle\aleph \mid \mathfrak{J}\rangle=\sum_{i}\langle\aleph \mid i\rangle\langle i \mid \mathfrak{J}\rangle .
$$

The transaction is complete if one of the buyers $\left(\mathrm{k}^{\text {th }}\right)$ bids an amount which is not topped up by any other auction participant and pays for the goods not more that the resignation price. This is now the player wins the auction. Buyers $1, \ldots \mathrm{k}-1(\mathrm{k} \leq \mathrm{N})$ drop out from the auction because having bid too low a price they fail to reach the final state $\Phi_{\text {out }}$ - in accordance with Pauli exclusion principle there is no free space for them there. The Pauli principle says that no two microobjects can be in the same state at the same time. The principle is responsible for the stability of matter. For auctions, it is responsible for an uninterrupted process.

Thus, a transaction really means a transition from the states of initial strategies adopted by participants

$$
|\Phi\rangle_{\text {in }} \text { to final state }|\Phi\rangle_{\text {out }}:|\Phi\rangle_{\text {in }}=\mathrm{T}|\Phi\rangle_{\text {out }}
$$

where

$$
\mathrm{T}=|\mathrm{p}\rangle_{\mathrm{in}}+\sum_{\mathrm{ij}}\left\langle\mathrm{q}_{\mathrm{i}} \mid \mathrm{i}\right\rangle\left\langle\mathrm{i}\left|\mathrm{T}_{\mathrm{A}}\right| \mathrm{j}\right\rangle\left\langle\mathrm{j} \mid \mathrm{q}_{\mathrm{j}}\right\rangle
$$

is transaction operator,

$|p\rangle_{\text {in }}$ - is the asking or reservation price,

$\mathrm{T}_{\mathrm{A}}$ - is an inter-state transition operator,

$\mathrm{q}_{\mathrm{i}}$ - is the price offered in bidding.

Buyers often lack the ability to put a specific value on an item of goods (specify the price at which they would give up the purchase). 
The price may evolve as a result of information continuously received by the buyer. This situation corresponds to the Heisenberg principle. For English auctions with numerous buyers, the Heisenberg principle may be formulated as follows: the product of two dispersion measures - purchase and sale - may not be smaller than Planck constant. Planck constant h corresponds to the lowest risk appetite of the player. This principle holds for any pair of players $(i, j)$, ipso ergo:

$$
\Delta \mathrm{q}_{\mathrm{i}} \Delta \mathrm{q}_{\mathrm{j}} \geq \mathrm{c} \cdot \mathrm{h} \quad(\mathrm{i} \neq \mathrm{j}, \mathrm{i}, \mathrm{j}=0,1, \ldots ., \mathrm{k})
$$

where $\mathrm{c}$ is a certain constant, $\mathrm{c} \geq 0$.

In addition, a rational strategy known from auction theory, which is not to bid a price above your own valuation: $\langle\mathrm{q} \mid \Phi\rangle_{\mathrm{i}} \leq\left\langle\mathrm{q} \mid \mathrm{v}_{\mathrm{i}}\right\rangle$, where $\mathrm{v}_{\mathrm{i}}$ is goods valuation of the $i^{\text {th }}$ bidder is also in line with the Heisenberg uncertainty principle.

Many more similar analogies can be found. At least one more deserves increased attention - the Schrödinger's cat paradox, as outlined in Section 3.3.

The seller's state $(\mathrm{k}=0)$ immediately before the transaction is closed (prior to measurement) can be expressed in the following form:

$$
\begin{gathered}
\left.\left|\Psi_{0}\right\rangle=\alpha \mid \text { transaction closed }\right\rangle+ \\
\beta \mid \text { transaction fails }\rangle
\end{gathered}
$$

where $|\alpha|^{2}+|\beta|^{2}=1$.

The state of the $\mathrm{k}^{\text {th }}(\mathrm{k}=1,2, \ldots \mathrm{N})$ buyer is characterised as follows:

$$
\begin{aligned}
& \left|\Psi_{\mathrm{k}}\right\rangle= \\
& \left.\quad \alpha_{1} \mid \text { satisfied with transaction }\right\rangle \otimes \\
& \quad \mid \text { transaction closed }\rangle+ \\
& \left.\beta_{1} \mid \text { uncomfortable with transaction failure }\right\rangle \otimes \\
& \quad \mid \text { transaction fails }\rangle
\end{aligned}
$$

where $\left|\alpha_{1}\right|^{2}+\left|\beta_{1}\right|^{2}=1$

It may be observed that the states described by formulae (28) and (29) correspond to 'diagnoses' of the external and internal observers in the Schrödinger's cat paradox. When an auction is completed the outcome state $\left|\Psi_{\mathrm{k}}\right\rangle_{\text {out }}$ is reduced to state: $\mid$ transaction completed \rangle or $\mid$ transaction fails $\rangle$, but no doubt the $\mathrm{k}^{\text {th }}$ buyer $(\mathrm{k}=$ $1, \ldots \mathrm{N})$ fees $\mid$ satisfied $\rangle$ when he/she succeeds in purchasing the goods on favourable terms or feels $\mid$ uncomfortable $\rangle$.

\section{Conclusion}

The modelling of economic phenomena based on quantum theory provides more precise descriptions than using the classical probability calculus. In addition, there are a number of analogies between quantum mechanics and social sciences. In both cases, finding the precise 'location' of an object requires taking numerous measurements (identifying waves of different frequencies).

This is the only way, with a high degree of uncertainty, to identify a more or less precise location of an object. A non-zero correlation the variables corresponding to specific economic phenomena proves their dependencies which may be compared to the concept of system entanglement in quantum theory. It is further understood that the evolution of a quantum system 'is determined' by the unitary operator affecting any given state at a point in time.

A similar role is played by the transaction operator in auction theory, as expressed in formula (17). The uncertainty and inaccuracy inherent to the description of a broad range of economic phenomena are not caused by the failure of statistical and forecasting methods but are inherent to such phenomena themselves.

A similar situation is found in quantum mechanics the complicated nature of phenomena plays a bigger role than the accuracy of measuring instruments. Consequently, both social and physical phenomena may only be described (identified) in approximative terms under the uncertainty principle.

A market described with the use of quantum mechanics tools is likely to multiply its closed transactions. For example, one can multiply profit by holding several auctions simultaneously. The Pauli exclusion principle does not prevent participation in other auctions. By applying a certain (well designed) strategy in a number of places at the same time does increase the probability of profit, which has recently been so evident in online auctions.

\section{References}

[1] Balicki A. - Analiza rynku (Market Analysis). Wydawnictwo Wyższej Szkoły Zarządzania, Gdańsk 2002. 
[2] Challet D., Marsili M., Zhang Y.C. - Modeling Market Mechanism with Minority Game. Physica A 276, 2000, pp. 284-315.

[3] Drabik E. - Aukcje $w$ teorii i praktyce (Auctions in Theory and Practice). Wydawnictwo SGGW, Warsaw 2007.

[4] Feynman R.P., Leigton R.B., Sands M. - Feynmana wyktady z fizyki (The Feynman Lectures on Physics). Vol. III. Mechanika kwantowa (Quantum mechanics). Państwowe Wydawnictwo Naukowe, Warsaw 1972.

[5] Krugman P., Obstfeld M. - Ekonomia międzynarodowa. Teoria i polityka (International Economics. Theory and Policy). Vol. 1, Wydawnictwo Naukowe PWN, Warsaw 2007.
[6] Moore D.S. - Analiza statystyczna danych doświadczalnych (Statistical Analysis of Experimental Data) [in] Współczesna matematyka. Dwanaście esejów (Contemporary Mathematics. Twelve Essays) (ed. Stern L.A.). Wydawnictwa Naukowo-Techniczne, Warsaw 1983, pp. 230-260.

[7] Mosetti G., Challet D., Zhang Y.C. - Heterogenous Time Scales in Minority Game. Physica A - 365, 2005, pp. 529-542.

[8] Piotrowski E.W. - Dwoistość wartości kapitału (Duality of Capital Value). Białystok 2001, preprint rePEc:sla:aekjkl:69PLv3.

[9] Schuster H.G. - Chaos deterministyczny. Wprowadzenie (Deterministic Chaos. Introduction). Wydawnictwo Naukowe PWN, Warszawa - Wrocław 1995. 